\title{
Development, Validation and Applications of the Monocyte Activation Test for Pyrogens Based on Human Whole Blood
}

\author{
Stefanie Schindler ${ }^{1,4}$, Sonja von Aulock ${ }^{1}$, Mardas Daneshian ${ }^{2}$ and Thomas Hartung 1,2,3 \\ ${ }^{1}$ Biochemical Pharmacology and ${ }^{2}$ CAAT-EU, University of Konstanz, Germany; ${ }^{3}$ CAAT, Johns Hopkins University, Bloomberg \\ School of Public Health, Baltimore, USA; ${ }^{4}$ current address: Foundation Animalfree Research, Zurich, Switzerland
}

\begin{abstract}
Summary
Microorganisms such as Gram-negative or Gram-positive bacteria, viruses and fungi contain components that activate the innate immune system. These components, called pyrogens (Greek: pyros=fire), can occur independently of viable microorganisms and are a major safety concern in parenterally administered drugs, since they can cause severe reactions such as fever, organ failure, and shock in the recipient. So far these drugs have been tested by injecting them intravenously into rabbits and measuring their fever reaction or, alternatively, by the Limulus Amoebocyte Lysate (LAL) test, employing the coagulation of the hemolymph lysate of Limulus polyphemus. Both tests have inherent limitations. A new in vitro monocyte activation test (MAT) based on human whole blood, capable of measuring all pyrogens relevant to the human patient, introduced in this journal in 1995, was validated and recently accepted by European Pharmacopoeia and US FDA. This review describes its principle, development, validation and the wide spectrum of applications, such as for testing of medical devices, blood products, toxic or immunomodulatory drugs, dialysis liquids, lipidic parenterals, and air quality. This alternative method promises to replace the rabbit pyrogen test fully and to overcome several limitations of the LAL assay.
\end{abstract}

Keywords: monocyte activation test, interleukin-1 $\beta$, validation study, alternatives to animals

\section{Introduction}

Pyrogens, as fever-inducing substances of microbial origin, can derive from dead or viable bacteria, viruses, or fungi and thus can occur even in sterile environments. Contaminations of parenterals with such substances can induce local or systemic inflammatory reactions intended to eliminate an invading pathogen in the recipient, including a rise in body temperature, but also more severe adverse reactions, such as shock, disseminated coagulation, organ failure, and even death. Therefore, the testing of parenterals prior to batch release is obligatory for manufacturers.

The best-known fever-inducing contaminant is a component of the cell wall of Gram-negative bacteria, i.e. endotoxin or lipopolysaccharide (LPS). Pyrogenic components of Grampositive bacteria are equally important and include lipoteichoic acid (LTA) (Morath et al., 2001) and peptidoglycan (Atkins and Morse, 1967; Rotta, 1975; Schleifer, 1975). Further possible pyrogenic contaminants are exotoxins (Watson, 1960), enterotoxins (Bodel and Atkins, 1965; Brunson and Watson, 1974), viruses (Atkins and Huang, 1958), and fungal components (Braude et al., 1960; Kobayashi and Friedman, 1964).

Received $8^{\text {th }}$ December 2009, accepted for publication $18^{\text {th }}$ December 2009
Classical pyrogen tests

Testing for pyrogens has been a major issue since the appearance of large volume parenterals in the 1930s. These bore a label claim of being pyrogen-free as assessed by the rabbit pyrogen test. This drew attention to the need for an official test procedure for non-pyrogenicity, which was strengthened by the heavy demand for large volume parenterals in World War II. A collaborative study was initiated to develop the rabbit pyrogen test (Welsh et al., 1943; McClosky et al., 1943), which led to the incorporation of the rabbit pyrogen test into various pharmacopoeias. Since then, all parenterals must be tested for pyrogens. This involves the measurement of the rabbit's body temperature after the application of not more than $10 \mathrm{ml} / \mathrm{kg}$ bodyweight of the substance to be tested. The very rigid fixation of the rabbit and the handling (injection) procedure can cause hyperthermia due to excitement and, therefore, lead to false-positive results. On the other hand, it has been reported that the fixation and lack of movement can cause hypothermia, yielding false-negative results (Grant, 1950). General limitations of animal tests have been discussed elsewhere (Hartung, 2008).

In 1964, Levin and Bang published that the hemolymph of the horseshoe crab Limulus polyphemus coagulates upon contact 
with endotoxin (Levin and Bang, 1964a,b). This led to the development of the Limulus amoebocyte lysate (LAL) test, which is employed to exclude endotoxin contamination in parenteral drugs. The Limulus is collected from beaches, its hemolymph is drawn out by puncture, and the animals are then thrown back into the sea. Ten to twenty percent do not survive the bleeding procedure (Rudloe and Hernkind, 1983; Thompson, 1998; Walls and Berkson, 2003). A recent study revealed that the mortality rate of the crabs subjected to biomedical bleeding is significantly increased compared to the unbled crabs (by $8.3 \%$ ), and the mortality is even higher when external stress factors like transport and holding methods are present (by 29.4\%) (Hurton et al., 2009).

The LAL has not been able to replace the rabbit test fully, since it is defined not as a pyrogen test, but as an endotoxin test, and fails to recognize, for example, Gram-positive or fungal contaminants, toxoids, or viral antigens. Given the perceived central role of Gram-negative endotoxin, it was nevertheless considered acceptable to substitute as a mere endotoxin test for most pharmacopoeial pyrogen testing. The fact that Gram-negative LPS has been identified and available for 50 years, and the LPS-specific LAL is available, has led to much research. The Gram-positive and fungal counterparts, meanwhile, have been largely neglected, although they comprise most spore-formers, which represent a key spread of pyrogens. Additionally, the LAL does not reveal the biological potency of a given endotoxin in the mammal, which can differ between bacterial strains by a factor of 10,000 (Fennrich et al., 1999; Dehus et al., 2006; Brandenburg et al., 2009). However, certain products that are tested in rabbits, such as various biologicals and vaccines, cannot be tested using the LAL.

\section{Fever reaction in the mammal}

The finding that mammalian immune cells produce an endogenous pyrogen when in contact with pyrogenic materials dates back to 1948 (Beeson, 1948). Bennett and Beeson identified leukocytes as the source of this factor in 1953. The nature of this substance was further elucidated by Dinarello et al. (1974), who identified two distinct proteins, probably the pro- and the mature form of interleukin $1 \beta$ (IL-1 $\beta$ ). The pyrogenicity of IL$1 \beta$, when injected at very low doses into rabbits, was proven by Dinarello et al. (1991). IL-6 and TNF- $\alpha$, which were isolated later, were found to be pyrogenic cytokines as well, though only at higher doses (Dinarello et al., 1986; Dinarello et al., 1991).

The current understanding of the mechanism of fever in the mammal, as reviewed by Dinarello (2004), is that these proinflammatory cytokines bind to receptors on the blood side of the organum vasculosum laminae terminalis (OVLT) and initiate the expression of the enzyme cyclooxygenase-2 (COX-2), which mediates prostaglandin (PG) $\mathrm{E}_{2}$ synthesis. Mice deficient in $\mathrm{COX}-2$ do not develop fever in response to injection with LPS, IL-1 $\beta$, or IL-6 (Li et al., 1999; Li et al., 2001; Li et al., 2003). Specifically one of altogether four $\mathrm{PGE}_{2}$ receptors in the brain, the EPR-3, is required to develop fever (Ushikubi et al., 1998; Dinarello et al., 1999), probably via the induction of a second messenger such as cAMP(Laburn et al., 1974). Thus, the pyrogenic cytokines cause a change in the set-point of body temperature in the hypothalamus and so mediate the fever reaction.
The finding that monocytes, a subfraction of the white blood cells, secrete proinflammatory cytokines such as IL- $1 \beta$ upon contact with pyrogenic material was the basis for the development of the whole blood test (Hartung and Wendel, 1995).

\section{Basic principle of the whole blood test}

\section{Blood incubation}

The procedure introduced 1995 (Hartung and Wendel, 1995) is described in detail by Hoffmann et al. (2005a) and Daneshian et al. (2009). Briefly, freshly drawn, heparinized human whole blood from a healthy donor is diluted in physiological, pyrogen-free clinical grade saline and brought together with the test sample. In response to pyrogens, the monocytes contained in the blood sample produce proinflammatory cytokines in a dosedependent manner. The proinflammatory cytokine IL- $1 \beta$ or IL-6 is measured by ELISA.

\section{ELISA procedure}

The IL-1 $\beta$ or IL- 6 in the sample is tyoically sandwiched between a monoclonal coat antibody and a polyclonal peroxidaselabeled detection antibody. Unbound material is removed by washing. The peroxidase metabolizes e.g. tetramethylbenzidine. The reaction is stopped with acid and the optical density (OD) is measured at $450 \mathrm{~nm}$.

\section{Controls}

As an assay control, a dose-response curve of an LPS e.g. from E. coli O111: B4 is performed in parallel in each assay. This LPS is calibrated to the international WHO reference standard from E. coli O113: H10 (Poole et al., 1997). The dose-response curve must contain the concentration $0.5 \mathrm{EU} / \mathrm{ml}$ and a negative control. The IL- $1 \beta$ released in response to the concentration of $0.5 \mathrm{EU} / \mathrm{ml}$ must test positive when compared to a negative control for the experiment to be valid. An endotoxin concentration of $0.5 \mathrm{EU} / \mathrm{ml}$ corresponds to $50 \mathrm{pg} / \mathrm{ml}$ of the international reference standard and is considered the threshold for fever in the most sensitive rabbit strains. This threshold was confirmed in a study performed at the Paul-Ehrlich Institute, which included 171 rabbits (Hoffman et al., 2005b).

\section{Testing for interference}

In order to test for interference of a given substance with the activity of the monocytes, samples (pure or diluted) are incubated together with a $0.5 \mathrm{EU} / \mathrm{ml}$ concentration of the LPS dose response curve. The mean OD of the spiked sample must be within a $50-200 \%$ range of the $0.5 \mathrm{EU} / \mathrm{ml}$ concentration of the dose response curve. If this is not the case, the sample has to be diluted until the interference criteria are met.

\section{Development of the Gram-positive standard lipoteichoic $\operatorname{acid}($ LTA)}

LTA from Staphylococcus aureus was first purified in a biologically active and endotoxin-free quality by Morath et al. (2001). Later, the improved purification procedure was applied to produce LTA from B. subtilis (Morath et al., 2002). The success- 
ful identification of the purified LTA as a pyrogenic substance, which is negative in the LAL (Morath et al., 2002; Draing et al., 2008) and therefore represents a pyrogenic principle that is only recognized by the rabbit pyrogen test and the cell-based assays, led to the inclusion of the Gram-positive standard derived from B. subtilis into the whole blood MAT (WBT) procedure. LTA of suitable quality has been prepared to produce an international reference material, for which a partner is currently sought.

In order to make this method commercially available and to replace the rabbit pyrogen test, the following steps were taken:

\section{Comparison of the in vitro reaction of human whole blood to that of rabbit whole blood}

Since the human whole blood test (WBT) aims to replace the rabbit pyrogen test, the sensitivity of both species towards different pyrogenic stimuli was compared using human and rabbit whole blood. For this, a rabbit whole blood test was developed, which followed the procedure of the human whole blood incubation in every detail (Schindler et al., 2003). Overall, the IL-1 $\beta$ response of the rabbits towards different pyrogenic stimuli was comparable to that of humans. Regarding the Gram-positive stimulus LTA, the rabbit blood was less sensitive than human blood, thus confirming the human whole blood test as an equal or even superior test system to reflect the human response.

\section{Establishment of the WBT as a test for biologicals}

Biologicals, such as protein solutions, cytokines, antibodies, heat shock proteins, blood coagulation factors, and vaccines for intravenous use pose a particular problem in pyrogen testing. They can influence the LAL results due to their characteristics, such as color and viscosity, and they are potentially immunogenic in the rabbit, causing fever reactions that are independent of contaminations. In any case, if immunogenic substances are tested, the animals may only be used once, which results in high costs for the manufacturers. The WBT does not pose such problems. Some examples of the application of the WBT for pyrogen testing of such samples are given below.

\section{Control of vaccines}

New batches of vaccines, released in 2001 against early summer meningoencephalitis, caused severe fever reactions in some recipients. Although they were negative in the LAL, these batches gave a high signal in the WBT. This phenomenon could be attributed to the omission of the mercury-containing additive thimerosal in these batches. The additive suppressed the IL- $1 \beta$ response in the WBT, making it likely that it also suppressed the pyrogenic property of the viral pyrogens in earlier batches (Fischer et al., 2001).

In 2003, Carlin and Viitanen demonstrated that trivalent vaccine (diphtheria, tetanus, and polio), which tested negative in the LAL, was a powerful inducer of IL-6 in 4 out of 8 donors in the whole blood incubation. This difference between the LAL and the WBT could be attributed to the toxoid of Corynebacterium diphteriae, and, to a lesser extent, to that of Clostridium tetani, both non-endotoxin pyrogens (Carlin and Viitanen, 2005). Additionally, in both studies, the authors found pronounced differences in the IL- 6 (and IL-1 $\beta$ ) response of different donors towards the vaccines and their components, although they displayed highly conserved LPS reactivity. This indicated a more variable inter-individual sensitivity of human donors towards these non-endotoxin stimuli. Nonetheless, it was demonstrated that pyrogenic reactions towards non-endotoxin stimuli can be just as vigorous as those towards endotoxin. These results show that for vaccines the rabbit pyrogen test cannot be replaced by the LAL and that only the measurement of the cytokine response of primary human cells, e.g. the WBT, represents an adequate alternative. Positive experiences with the WBT assay were also reported for Neisseria meningitidis vaccines (Stoddard et al., 2009).

Measurement of albumins and other proteins for injection Pyrogenic reactions of human patients after the administration of human serum albumin, which had tested negative in the rabbit, were observed in 1978 (Steere et al., 1978). In this study, the LAL yielded positive results without perceivable patient reactions. Pool et al. (1998) tested 22 batches of human serum albumin (HSA), fibronectin, and stabilized human serum solutions using artificial contaminations of endotoxin and LTA from $B$. subtilis. None of these products interfered with the production of IL- 6 by whole blood, whereas one batch of artificially contaminated albumins tested false-negative in the LAL. Another study using the human whole blood test performed with albumins, coagulation factor, vaccines, and immunoglobulins indicated a high sensitivity and reliability of the WBT for these substances (Fennrich et al., 1999).

A comparison between the rabbit and the human whole blood test for the detection of pyrogens in albumins was performed by Spreitzer et al. (2002) with 29 batches of human serum albumin. The WBT was clearly superior to the rabbit test, especially at the limit of detection of $5 \mathrm{EU} / \mathrm{kg}(0.5 \mathrm{EU} / 10 \mathrm{ml} / \mathrm{kg})$, with the WBT retrieving all 29 spiked samples as positive compared to only 5 positive rabbit tests, one false negative and 23 temperature rises that would have required a repetition of the test. This limit of detection represents the $0.5 \mathrm{EU} / \mathrm{ml}$ pyrogenic threshold.

\section{Validation}

Six cell-based assays, including two variants of the WBT measuring IL-1 and IL-6, respectively, were validated in an international collaborative study including laboratories from Austria, Germany, Switzerland, England, Norway, and Italy with the participation of several National control institutions. The study examin assays such as the cell line THP-1 with the endpoint TNF- $\alpha$ (Eperon and Jungi, 1996), or with the endpoint neopterin (Werner-Felmayer et al., 1995; Peterbauer and Werner-Felmayer, 1999), the cell line Monomac-6 measuring IL-6 (ZieglerHeitbrock et al., 1988), isolated peripheral blood mononuclear cells (PBMCs) with endpoint IL-6, and the human whole blood 
test (Hartung and Wendel, 1995), using blinded endotoxin stimuli and altogether 13 intravenously applied drugs. Sensitivities ranged between $73-96 \%$ and specificities between $90-97 \%$. The WBT measuring IL-1 achieved 73 and 93\%, and the WBT IL-6 88.9 and $96.6 \%$, respectively. The development and outcome of this study is described in detail elsewhere (Hartung et al., 2001; Hartung, 2002; Hoffmann et al., 2005a).

\section{Development of the commercially available WBT kits}

The established WBT procedure was adapted to materials provided by Charles River Endosafe and a commercially available kit was developed, which was named In Vitro Pyrogen Test (IPT). This kit contains all the reagents necessary for the incubation and ELISA procedure except the human whole blood. The kit was produced under a license until September 2008. The Biotest microbiology unit (hycon@biotest.de) is working on a new commercially available kit of the patented WBT procedure. The development of this in vitro kit under the name of PyroDetectSystem containing all necessary components is in a final stage. First test kits for customer trial will be available in the beginning of 2010 .

\section{Development of cryopreserved blood}

Fresh human whole blood is a highly perishable item that cannot be stored longer than 4 hours at room temperature without loss of sensitivity for pyrogens. Additionally, it is not easily available, a potential hazard due to unrecognized infections (HIV, hepatitis) and, due to donor differences, not standardizable. In order to overcome these difficulties a procedure was developed to successfully freeze and store whole blood. The protocol closely followed the method of de Boer et al., 1981, who had already successfully frozen isolated monocytes for other purposes. Blood from five healthy donors is mixed with $10 \%$ endotoxin-free dimethylsulfoxide (DMSO) (v/v ratio) and left to stand for 15 minutes. The blood is then pooled (Fig. 1) and frozen in a computer-controlled freezing process to $-120^{\circ} \mathrm{C}$. The blood is stored in the vapor phase of liquid nitrogen and, after thawing, can be used like fresh blood without any washing steps. The cryopreserved pooled blood renders highly reproducible results and is at least equal to fresh blood concerning a wide variety of applications and stimuli (Schindler et al., 2004).

\section{Validation of the cryopreserved blood}

In an additional validation process, which followed the exact procedure of the former process described above, both methods of cryopreservation were validated (Schindler et al., 2006a). Furthermore, the WBT incubation steps, which had been developed and validated in pyrogen-free reaction tubes, had, in the meantime, been successfully transferred to the 96-well microtiter plate by reducing the volumes used and adapting the protocol accordingly, and the fresh blood incubation in the plate was validated, as was the use of both versions of the cryopreserved blood in the 96-well microtiter plate. The overall performance of all approaches was very good, with sensitivities of over $90 \%$ and specificities around $80 \%$. Remarkably, these excellent performance characteristics were achieved although the spike concentrations were chosen at and below the defined pyrogenicity threshold of 0.5 EU/ml (Hoffmann et al., 2005b). Indeed, misclassifications only occurred for these borderline cases. Therefore, the WBT could be improved concerning its availability, its performance and its handling (Tab. 1). The method has been

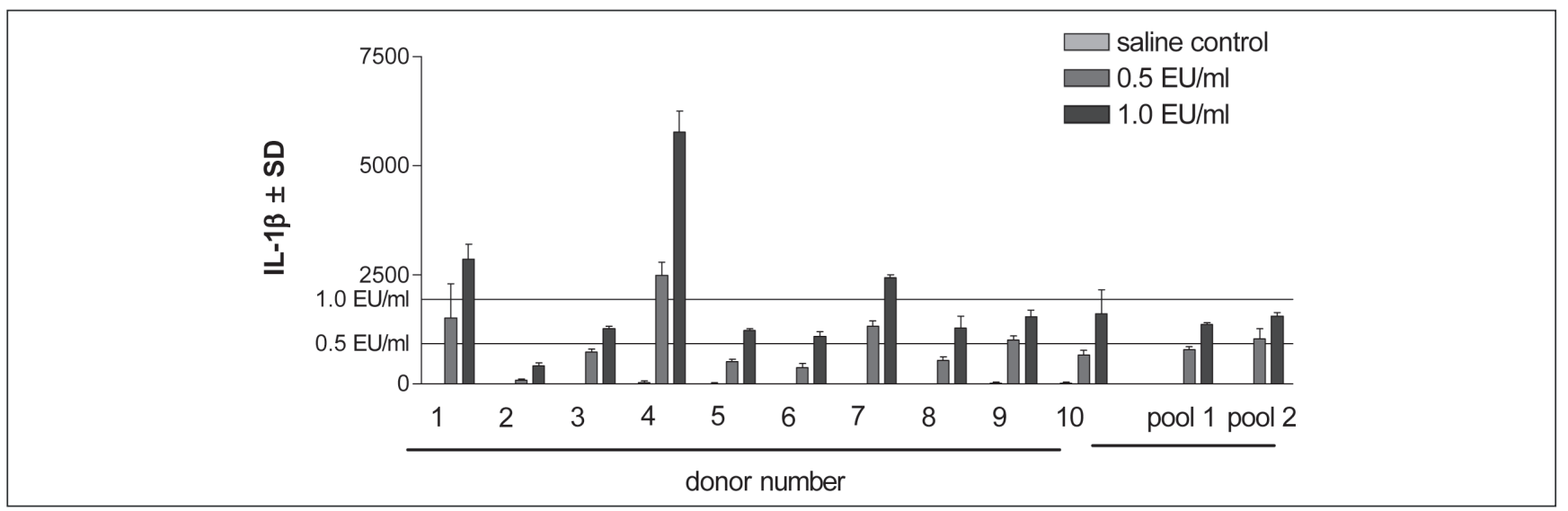

Fig. 1: Comparison of the reactivity of frozen blood from 10 individual donors and that of pooled blood from the same donors The calculated means of the response towards the $0.5 \mathrm{EU} / \mathrm{ml}$ stimulus of the five individual donors corresponds to the actual $0.5 \mathrm{EU} / \mathrm{ml}$ response of the pool blood. The higher response of donor 4 is therefore levelled out. Pool 1: The blood was pooled before freezing Pool 2: The blood was pooled after thawing Since blood frozen using the described method could only be stored and shipped in the vapor phase of liquid nitrogen - a reagent that is not available to all laboratories - an alternative freezing method was developed by the Paul-Ehrlich Institute, Langen, Germany (Spreitzer et al., 2008). The alternative cryopreservation method provided a blood that could be stored (and shipped) at -40 to $-80^{\circ} \mathrm{C}$, therefore making the blood available for users without liquid nitrogen infrastructure. 
internationally validated by the European Centre for the Validation of Alternative Methods in 2006 and the US Interagency Coordinating Committee for the Validation of Alternative Methods in 2008. European Pharmacopoeia accepted the method in March 2009 (ALTEX News, 2009, 26, 143). An FDA statement of acceptance was sent to ICCVAM in April 2009 (http://iccvam.niehs.nih.gov/methods/pyrogen/transmitNov08/FDAResponse.pdf). The WBT has also been included in the draft ISO guideline for biological testing of medical devices (ISO/TC 194, working group pyrogen testing).

\section{Special adaptations}

\section{Medical devices}

Due to manufacturing and handling, medical devices can bear pyrogens on their surface when brought into the human organism, leading to inflammatory reactions and reduced biocompatibility. Recognizing this problem, the Medical Device Directive 93/42 EEC states that medical devices must be designed and manufactured in such a way that they will not compromise the clinical condition or the safety of the patients. The Association for the Advancement of Medical Instrumentation (AAMI) stated in 2001 that products with direct or indirect contact with the circulation system or the lymph, or which interact systemically with the body, should be tested for pyrogens (AAMI, 2001).

Products with direct (e.g. blood bags, needles) and indirect (e.g. swabs, gloves) contact to the blood circulation can have serious impact on the organism, as contaminations induce systemic reactions. A severe case of contact dermatitis due to endotoxin contamination of surgical gloves was described in 1984 by Shmunes and Darby. After eight pyrogenic reactions in 69 patients undergoing heart catheterization, Kure et al. described endotoxin contamination of extracts of the hospital's surgeon's latex gloves, which evoked fever in rabbits and could be successfully transmitted to cardiac catheters (Kure et al., 1982). Grötsch et al. were able to evoke fever reactions in rabbits with an eluate of gloves containing up to $2560 \mathrm{EU}$ (Grötsch et al., 1992).

However, medical devices pose a particular problem for pyrogen testing, since they cannot be examined directly with the rabbit or the LAL test. Their diversity with regard to size, form, material, and mode of application challenges the existing assays, demanding individual approaches. In order to judge a possible contamination, an eluate of the respective material must be either injected into the rabbit or used in the LAL. However, it is unclear how well rinsing a medical device in water can release pyrogens from its surface, and the dilution of such released pyrogens in a large volume of rinsing water also reduces the limit of detection. For LTA, it has been shown that surface presentation increases potency about 1,000-fold (Deininger et al., 2008), raising serious doubts about the value of eluates. The alternative of transplanting the questionable material directly into the rabbit is highly invasive, causing possible reactions not associated with pyrogenic contaminations but rather with tissue damage, and is therefore questionable in its ethical and scientific implications. The obvious advantage of the WBT over the classical test methods is that the whole blood comes into direct contact with the respective device and no preparation of an eluate is required (Hasiwa et al., 2007). This has been demonstrated using aneurysm clips as proof of principle (Mazzotti et al., 2007). Additionally, unlike the LAL, the WBT detects all pyrogens relevant to humans, not only endotoxin. It has also recently been successfully adapted to eye lenses (Wener et al., 2009).

Testing for the inflammation-inducing potential of implant

Tab. 1: Outcome of the validation of the basic WBT procedure using reaction tubes and fresh blood and of the WBT methods using cryopreserved or fresh blood in a microtiter plate

Results of the International catch-up validation study (Schindler et al., 2006a). The rabbit data were modeled

(Hoffmann et al., 2005a) based on variability between individual animals at the given endotoxin spike concentration (Hoffmann et al., 2005b) assuming no interference.

\begin{tabular}{|c|c|c|c|c|c|}
\hline Test & $\begin{array}{l}\text { Inter-laboratory } \\
\text { reproducibility (\%) }\end{array}$ & $\begin{array}{l}\text { Sample size: } \\
\text { sensitivity }\end{array}$ & Sensitivity (\%) & $\begin{array}{l}\text { Sample size: } \\
\text { specificity }\end{array}$ & Specificity (\%) \\
\hline $\begin{array}{l}\text { WBT } \\
\text { Fresh blood } \\
\text { Reaction tubes }\end{array}$ & $\begin{array}{l}\text { DL-NL1: } 72.9 \\
\text { DL-NL2: } 81.6 \\
\text { NL1-NL2: } 70.2\end{array}$ & 88 & 72.7 & 59 & 93.2 \\
\hline $\begin{array}{l}\text { WBT } \\
\text { Cryopreserved } \\
\text { blood }\left(-80^{\circ} \mathrm{C}\right)\end{array}$ & $\begin{array}{l}\text { DL-NL 1: } 86.7 \\
\text { DL-NL 2: } 87.5 \\
\text { NL 1-NL 2: } 100\end{array}$ & 77 & 97.4 & 45 & 82.2 \\
\hline $\begin{array}{l}\text { WBT } \\
\text { Cryopreserved } \\
\text { blood (nitrogen) }\end{array}$ & $\begin{array}{l}\text { DL-NL 1: } 66.0 \\
\text { DL-NL 2: } 63.3 \\
\text { NL 1-NL 2: } 83.3\end{array}$ & 74 & 82.4 & 46 & 89.1 \\
\hline $\begin{array}{l}\text { WBT } \\
\text { Fresh blood } \\
\text { plate }\end{array}$ & $\begin{array}{l}\text { DL-NL 1: } 88.1 \\
\text { DL-NL 2: } 89.7 \\
\text { NL 1-NL 2: } 91.5\end{array}$ & 84 & 98.8 & 55 & 83.6 \\
\hline $\begin{array}{l}\text { Rabbit } \\
\text { (theoretical) }\end{array}$ & & & $88.3 \%$ & & $57.9 \%$ \\
\hline
\end{tabular}


surfaces for the judgment of biocompatibility is a relatively new field. In the early 1980s, it was noted that the monocyte is one of the first cells to arrive at an implant site and displays manifold functions (for review see Anderson and Miller, 1984; Ziats et al., 1988). Its specific preference for rough and hydrophobic surfaces differs from that of fibroblasts (Rich and Harris, 1981). The role of cytokine production of the monocytes/macrophages in the early stages of implant insertion were, until now, poorly understood. The fact that some materials are obviously capable of modulating the cytokine response (Soskolne et al., 2002; Refai et al., 2004) makes it difficult to distinguish a genuine pyrogenic contamination from an unspecific activation and poses the problem of adequate negative controls. For this purpose a model was developed for the thorough depyrogenation and testing of metallic or plastic surfaces with the WBT in order to gain experiences about possible inherent activating or inhibiting characteristics of materials (Kullmann, 2002). The study showed that pyrogenic contaminations on surfaces can be reliably removed only when heated for $5 \mathrm{~h}$ at $300^{\circ} \mathrm{C}$. This applied to titanium, titanium alloy $\left(\mathrm{TiAl}_{6} \mathrm{~V}_{4}\right)$, and steel material for implants. The blood was incubated directly in a depyrogenized microtiter plate with contact to the surface to be tested. Artificial contaminations were recognized in a dose-dependent manner.

Some medical devices are absorbed completely by the body, as are any contained pyrogenic contaminations. Examples are liposomes and alginate microcapsules as carriers of drugs. With the latter, the solutions could be clearly identified as positive or negative (Fig. 2).

\section{Cellular therapeutics}

Cellular therapeutics have been defined as living cells which are transferred into the intact organism in order to introduce a new

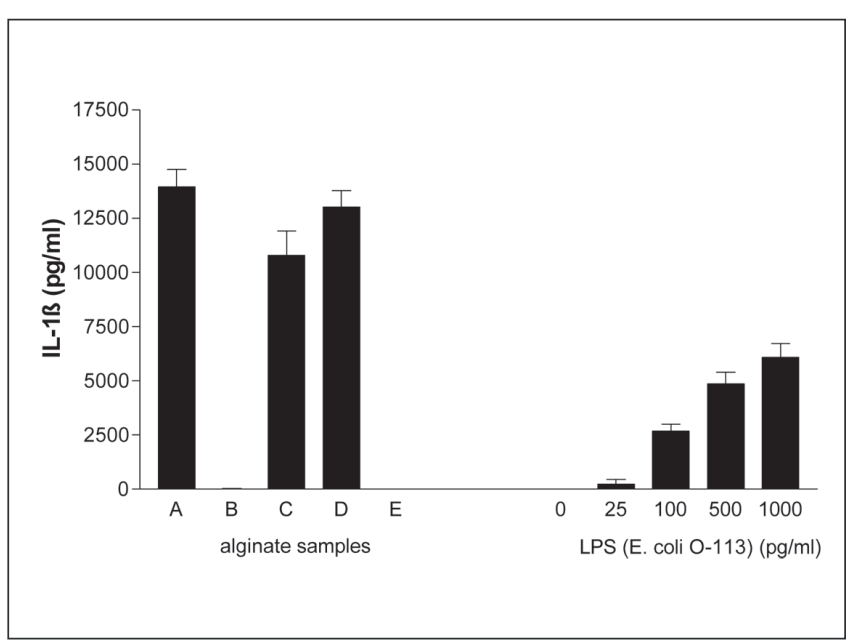

Fig. 2: IL-1 $\beta$ production of fresh blood upon stimulation with different alginate solution samples (A-E)

Several production lots of commercial alginates for clinical use were tested and compared to a reference LPS concentration response curve. Clean and contaminated samples could be clearly distinguished. The product cannot be tested in the LAL due to false-positive results. function or restitute a defective one (Rosenberg, 2001). This includes a wide variety of cells such as chondrocytes, stem cells, bone marrow cells, and blood cells such as lymphocytes, erythrocytes, and thrombocytes. The latter pose a particular problem since they are stored at room temperature and are, therefore, easily subject to extensive bacterial growth. Transfusion reactions ranged from rigors, fever, and chills, all the way to septic shock. The problem is under- rather than overrated, since numerous clinical events are not recognized as being transfusionassociated but are attributed, rather, to the underlying disease. Additionally, medication and immunosuppression might mask an existing septic/pyrogenic event. Recently, attention has focused on viral infections, although the incidence in blood products is less than 1 in 1,000,000 per unit for HIV ( 1 in 3,000 for bacterial contaminations) (Blajchman et al., 2005). Two large studies in France (BACTHEM study, Perez et al., 2001) and the USA (BaCon study, Kuehnert et al., 2001) revealed that platelets hold a significantly higher risk of bacterial contamination than RBCs, irrespective of their being single-donor or pooled preparations. Pathogens associated with bacteremia in the US study were 59\% Gram+ (mainly skin contaminants such as Staphylococcus and Streptococcus spp, and Propioni bacteria) and $41 \%$ Gram-negative (Coli, Serratia, Enterobacter). Yersinia enterocolitica was not found, although it occurs frequently in transfusion-related sepsis and was responsible for 7 of the 8 recorded fatalities in the US 1986-91.

Incidences increased with prolonged storage, and both studies linked fatalities to the occurrence of Gram negative bacteria. The US study determined endotoxin levels as well (up to $273,500 \mathrm{EU} / \mathrm{ml}$, according to LAL). The authors estimated rates of transfusion-transmitted bacterial infections of 1:100,000 for platelets and 1 in 5 million for RBC, with fatalities of 1 in 500,000 and 1 in 8 million, respectively. All in all, Gram-negative bacteria tended to occur more frequently in RBCs, probably due to the storage conditions.

In July 2004, a roundtable meeting on bacterial detection, held during the Annual Congress of the International Society of Blood Transfusion in Edinburgh, discussed the limitations of current testing methods. Currently, culturing methods such as the very sensitive BacT/ALERT method are considered the best, though time-consuming. (12h to 7 days). Concentrates are released on a "negative-to-date" basis and recalled if necessary. The panel reported occurrences in the Netherlands, where platelet concentrates containing skin contaminants were tested positive only after $48 \mathrm{~h}$. By this time, the batch had been released and about $50 \%$ of the units had already been transfused. Very similar events were described by Belgian blood centers (Blajchman et al., 2005). Additionally, anaerobic bacteria (Corynebacterium spp.) are picked up later, and there is extra cost involved. However, anaerobic bacteria have been reported in fatal septic transfusion incidents (McDonald et al., 1998). Altogether, culture methods are incapable of providing complete safety, and other, especially quicker methods, are sought. An inactivation method, the photochemical treatment (PCT) (Wollowitz, 2001), has been developed. Still, it must be kept in mind that this inactivation will inhibit growth but have no influence on the already existing pyrogenic content. Therefore, the testing of these cells and 
their suspension materials is an interesting future challenge for the WBT. Pretesting of clinical grade erythrocytes and thrombocytes intended for transfusion indicated interference-free retrieval of an artificial endotoxin spike (Fig. 3), when compared to the saline control.

\section{Dialysis}

Pyrogenic reactions in hemodialysis (HD) patients at the end of a session were first associated with high bacterial and endotoxin levels by Raij et al. (1973) and Favero et al. (1974). Since then, contaminations have been found in the pure water (Klein et al., 1990; Pegues et al., 1992; Kulander et al., 1993; Bambauer et al., 1994), the machines, especially to areas with low circulation or dead spaces, which serve as a reservoir for bacteria (Phillips et al., 1994), filter materials (Schouten et al., 2000), and bicarbonate concentrates (Pegues et al., 1992).

In 1993, the AAMI released recommendations for the quality of treated water and dialysate, which restricted the content of heterotrophic bacteria to 200 and $2000 \mathrm{cfu} / \mathrm{ml}$, respectively. Studies in Germany (Bambauer et al., 1994), Greece (Arvanitidou et al., 1998), the USA (Klein et al., 1990), and Canada (Laurence and Lapierre, 1995) revealed that even these moderate standards are not met, which is even more critical considering that a patient with chronic renal failure receives up to 4001 of dialysis fluid a week. Next to Gram-negative bacteria, cocci (micrococci, staphylococci, and streptococci) were found in the dialysate of 83, 70, and $10 \%$ of the centers, respectively, indicating the importance of Gram-positive contaminations. That this might indeed be crucial for judging the pyrogenic load for a dialysis patient was assessed by Marion-Ferey et al., who tested scrapings of bacterial biofilms in dialysis tubes and found a 20-fold higher response in the WBT than in the LAL (Marion-Ferey et al., 2005). The passage of cytokine-inducing substances, not only endotoxins, but exotoxins and peptidoglycans as well, through the dialysis membrane has been demonstrated (Evans and Holmes, 1991; Lonnemann et al.,
1992; Urena et al., 1992; Tsuchida et al., 1997).

The exposure of HD patients to pyrogens is thought to contribute to inflammatory processes in the joints and bones and therefore to the carpal tunnel syndrome and arthropathy associated with long-term HD (Dinarello et al., 1988; Miyasaka et al., 1992). In 1991, Baz et al. showed that the use of ultrapure water delays the onset of the carpal tunnel syndrome. The group of Schwalbe (1997) showed in a retrospective study that the incidence of amyloidosis decreased between 1988 and 1996, coincident with the introduction of reverse osmosis, a very effective method for purifying water. A connection with other phenomena such as malnutrition, poor immune responses, and high incidence of malignant tumors in long-term HD patients and cytokine production has yet to be established.

All in all, the testing for pyrogens in dialysis fluids is a crucial issue for the safety of patients. Since the fluids themselves are either highly hyper- or hypotonic, a variant for testing dialysis fluids in the WBT established the percentages of diluents and samples that can be tested (Daneshiau et al., 2008). Still, the problem remains that the patients receive very high volumes of fluid in one session, and therefore pyrogens must be detected at very low concentrations. A promising possibility is a modification of the basic WBT protocol, the so-called adsorb and wash IPT (AWIPT), discussed later, which can concentrate pyrogens on the surface of albumin-coated macroporous beads, thus greatly enhancing sensitivity (Daneshian et al., 2008).

\section{Airborne pyrogens}

Inhalable whole or fragments of microorganisms (organic dust, OD) have long been recognized as causes of airway hyperreactivity. The most prominent illness related to inhalation of $\mathrm{OD}$, i.e. hypersensitivity pneumonitis or extrinsic allergic alveolitis, was first described in Iceland in 1874 and termed heykatarr. These involve type III reaction ("immune complex disease") as well as type IV hypersensitivity (antibody independent) reaction.

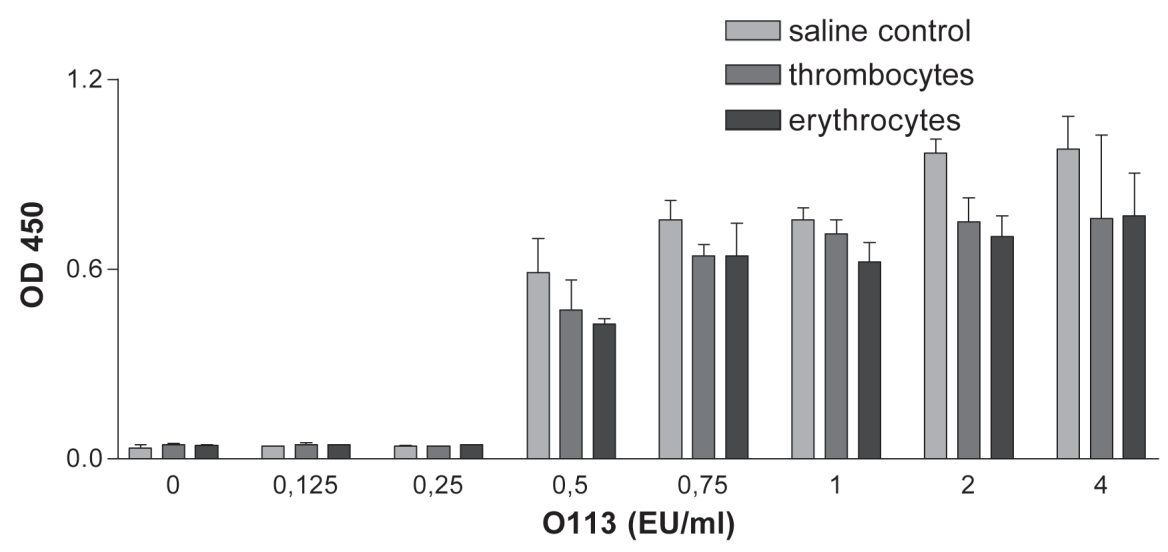

Fig. 3: Retrieval of endotoxin spikes in red blood cell and platelet concentrates

Clinical erythrocyte and platelet concentrates were spiked with reference LPS at the concentrations indicated. WBT was carried out directly on these samples. 
Here, proinflammatory cytokines and chemokines activate alveolar macrophages, leading to a migration of $\mathrm{CD} 8^{+}$lymphocytes into the lung tissue, causing granuloma formation, and promoting the development of pulmonary fibrosis (Mohr, 2004). There are approximately 300 etiologies of hypersensitivity pneumonitis associated with workplace-specific exposure (particularly to mold species), e.g. farmer's lung, cheese worker's lung, and bagassosis. The direct stimulation by pyrogenic components appears to contribute to these inflammatory processes.

Monday sickness (or Monday fever), with its typical symptoms (chest tightness, respiratory distress, and coughing), was described as early as 1936 (Prausnitz, 1936) and associated with endotoxins by Baenkler in 1999. In 1942, rural mattress makers experienced headache, nausea, chills, and fever about 6 hours after exposure to low-grade cotton. Neal et al. associated these phenomena with high numbers of Gram-negative bacteria in the material (Neal et al., 1942). Additionally, milder symptoms occurring 8 hours after exposure could also be evoked by sterilized cotton, which was thought to be due to remaining endotoxin. A highly significant $(\mathrm{r}>0.95)$ dose-response relationship between Gram-negative bacteria count and symptoms of byssinosis, such as chest tightness, airway inflammation, and coughs, was established by Cinkotai et al. (1977). In the same study, a good correlation existed between symptoms and mainly Gram-positive bacteria, whereas one to fungal spores could not be established. Acute bronchoconstriction, as well as chronic airway irritation with bronchitis and decrements in airflow over the work day, have been reported for personnel working in animal confinement buildings (e. g. Donham et al., 1984a,b; Thelin et al., 1984).

Long term consequences are of allergic, inflammatory, and immunostimulatory nature, e.g. organic dust toxic syndrome (ODTS) and chronic bronchitis. The LAL test for these contaminants has the drawback that it does not reflect the biological potency of a given LPS in the mammal (Hartung et al., 2000) and the LAL test can only be performed with an eluate of a filter or by impingement, i.e. the air to be tested is led through pyrogen-free water which is then tested in the LAL. The higher pyrogen retrieval by impingement when compared to filtration, possibly due to the incomplete eluation of the sample from the filters, was demonstrated by Zucker et al. (2000). A new approach of measuring the integral inflammatory activity in air samples in different environments by WBT was reported by Kindinger et al., 2005. A defined amount of air is drawn through a filter in a sealable plastic monitor. The blood incubation is performed directly on the filter inside the monitor, thus making any handling of the filter unnecessary. When compared to the LAL, a 2 to 25 -fold higher pyrogenic load was found in the WBT in samples drawn in parallel. Epidemiological studies will show what levels of exposure to inflammatory stimuli in the air eventually lead to the above-mentioned lung diseases. The method has been further standardized (Liebers et al., 2009).

\section{Lipidic formulations}

In January 2004, pyrogen testing of so-called small volume parenterals $(<15 \mathrm{ml})$ became obligatory in Europe. This concerns many formulations that had not been subjected to pyrogen testing before, such as vitamin preparations and steroids. Many of these are applied intramuscularly or subcutaneously and are therefore not necessarily of a hydrophilic nature. This poses a completely new challenge to all methods of pyrogen testing, since a lipophilic substance on the one hand cannot be injected intravenously into the rabbit due to the danger of clogging small vessels with lipid drops and severely damaging vital organs and will, on the other hand, influence the OD measured in the LAL due to the formation of oil droplets. Furthermore, the pyrogenic portion of LPS, lipid A (for review see Rietschel et al., 1993) has been reported to be masked by lipoproteins (Emancipator et al., 1992) and lipophilic parenterals (Paulssen and Michaelsen, 1984) in the LAL.

Therefore, the WBT procedure was adapted to suit lipophilic substances. As a first step, interference-free oils, such as sesame oil, were identified by comparing an LPS dose response curve in these oils with a similar curve done in saline. Surprisingly, many oils (sesame oil, peanut oil, paraffin, and miglyol) were interference-free, while others interfered strongly by suppressing the endotoxin stimulus added. Oils which proved interference-free were then used as diluents for interfering end-products. It was possible to dilute the interference to non-detectable limits with full recovery of an artificial endotoxin spike. From this minimum valid dilution a possibly detectable endotoxin concentration could be calculated, which was $20 \mathrm{EU} / \mathrm{ml}$ for the respective endproducts. Since these products are applied at a very small volume ( $1 \mathrm{ml}$ per person) a relatively high endotoxin concentration can be tolerated, which will predictably not pose a health hazard for the recipient. The established protocol leaves a broad safety margin, especially since the criteria for intravenous drugs were applied (Schindler et al., 2006b).

\section{AWIPT and AcWIPT (absorb and wash IPT as well as accumulation and wash IPT)}

Another interesting development is the so-called absorb and wash IPT (AWIPT). It uses macroporous acrylic beads with immobilized human serum albumin (HSA), which has a higher affinity than native plasma albumin to endotoxin (Zimmermann et al., 1999). These beads were originally developed for the extracorporeal apheresis of endotoxin in the early hyper-inflammatory phase of Gram-negative sepsis patients. The AWIPT uses this material to collect the endotoxin contained in a sample after an absorption phase in the substance to be tested. The beads are then washed in order to remove the interfering (unbound) material and can then be used directly in the IPT incubation. It could be shown that this works also for the LTA of Staphylococcus aureus. This procedure has already brought promising results with substances which cannot be examined in the rabbit pyrogen test and interfere with the LAL and the unmodified WBT procedure, i.e. toxic or immunomodulatory drugs such as taxol, gentamicin, or tobramycin (Daneshian et al., 2006). With the inclusion of the adsorption and washing step into the WBT procedure, the detection of pyrogenic contaminations in these sample drugs improved without decreasing the sensitivity of the IPT $(25 \mathrm{pg} / \mathrm{ml})$.

The HSA-bead adaptation also allows the testing of highvolume parenterals, where even very low concentrations of endotoxin, i.e. below the detection limit of current pyrogen tests, may sum up to relevant quantities that induce pyrogenic responses. Dialysis patients, who are exposed to hundreds of 
litres of fluids every week, pose a prime example. To them, the dialysis membrane is the only barrier between the microorganisms and pyrogens in the dialysis fluid and the blood stream. A variety of accessory symptoms associated with the enrichment of pyrogenic impurities in dialysis patients are thought to lead to dialysis related chronic inflammatory disease, e.g. increase of soluble CD14, elevated C-reactive protein, fibrinogen, amyloid protein, reduced transferrin, and albumin (Mitzner et al., 1995; Amato, 2002; Tranaeus et al., 2008). The testing of the efficiency of these membranes in holding back pyrogens is, in turn, dependent on the detection limits of our current pyrogen test systems. The maximum pyrogen concentrations of dialysis fluids and water is suggested by various pharmacopoeias and the AAMI (Association for the Advancement of Medical Instrumentation), and the ranges are between 0.03 (ultrapure) and 2 $\mathrm{EU} / \mathrm{ml}$ (Ward, 2004). These limits have also been defined based on the sensitivity of the LAL.

The accumulation wash IPT (AcWIPT) uses the HSA-beads to collect the endotoxin from solutions containing low concentrations of endotoxin on their surface until a contamination can be detected. Using this procedure, the detection limit of the WBT could be lowered (from $0.25 \mathrm{EU} / \mathrm{ml}$ of $E$. coli endotoxin down to $0.001 \mathrm{EU} / \mathrm{ml}$ ) (Daneshian et al., 2008), which is 10-fold more sensitive than the most sensitive LAL test available (Fig. 4). As the structure of some pyrogens may not yet be known, the spectrum of pyrogens that are detectable with these beads might be limited. However, the HSA-beads were able to collect the prominent pyrogens LPS, LTA, and the fungal pyrogen zymosan in both described procedures, i.e. AWIPT and AcWIPT.

All in all, the further development of the WBT into the modified forms of the AWIPT and AcWIPT promises to overcome shortcomings due to interferences of given drugs or substances with the classical WBT procedure, to lower the detection limit by $\log$ orders, and to provide a useful tool for the testing of toxic or strongly interfering substances, even those that suppress the immune system and therefore cytokine production.

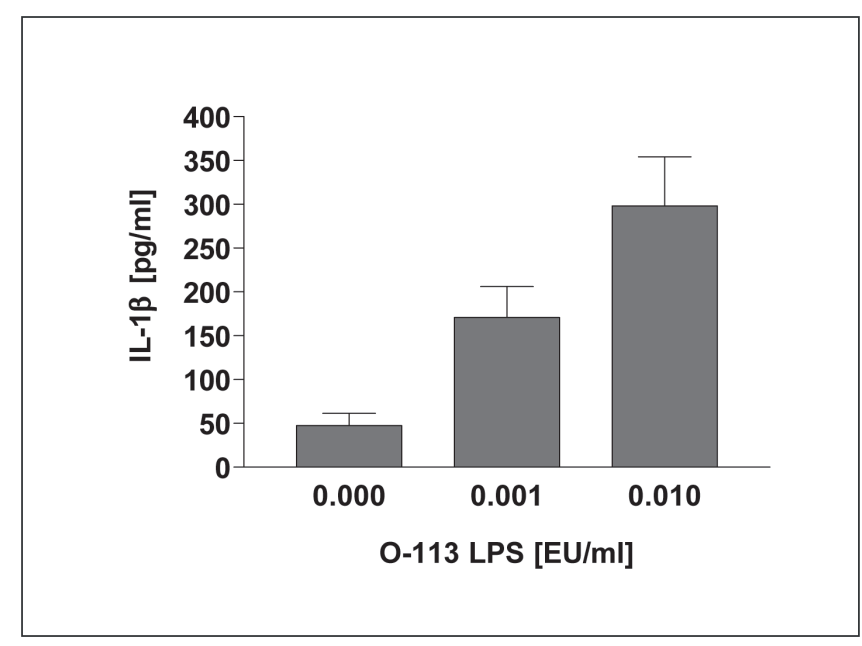

Fig. 4: Limit of detection in the AcWIPT

By accumulating endotoxin to human serum albumin coated beads and measuring these in the WBT (Daneshian et al., 2008), the limit of detection can be lowered by several log orders.

\section{Conclusions}

Pyrogens (fever-inducing substances) from microorganisms can occur as contaminations of parenterals. Until now, the safety of injectable drugs has been assessed by the in vivo rabbit pyrogen test and the in vitro Limulus amoebocyte lysate test (LAL).

The new cell-based in vitro pyrogen test based on fresh or cryopreserved human whole blood (WBT) has been successfully validated and has proven to be a reliable and useful tool for a wide spectrum of applications, ensuring patient safety in many medical fields such as hydrophilic and lipophilic drugs, dialysis fluids, airborne pyrogens, medical devices, and biologicals. It is capable of measuring all known pyrogens relevant for the human and is highly reliable, robust, and easy to perform.

\section{Abbreviations}

AAMI: Association for the Advancement of Medical Instrumentation

AcWIPT: accumulation and wash in vitro pyrogen test

AWIPT: absorb and wash in vitro pyrogen test

CAMP: cyclic adenosinmonophosphate

DMSO: dimethylsulfoxide

ECVAM: European Centre for the Validation of Alternative

Methods

ELISA: enzyme-linked immunosorbent assay

ELC: endotoxin limit concentration

EU: endotoxin unit

HD: hemodialysis

HAS: human serum albumin

IL: interleukin

IPT: in vitro pyrogen test

LAL: Limulus amoebocyte lysate

LPS: lipopolysaccharide

LTA: lipoteichoic acid

NIH: National Institutes of Health

NIBSC: National Institute of Biological Standards and

Controls

OD: optical density

OVLT: organum vasculosum laminae terminalis

PBMCs: peripheral blood mononuclear cells

PEI: Paul-Ehrlich Institute;

PG: prostaglandin

POD: peroxidase

RNA: ribonucleic acid

TMB: tetramethylbenzidine

TNF: tumor necrosis factor

USP: United States Pharmacopoeia

WBT: whole blood test

\section{References}

Amato, R. L. (2002). The link between dialysate and chronic inflammatory disease in hemodialysis. Nephrol. Nurs. J. 29, 490-4922.

Anderson, J. M. and Miller, K. M. (1984). Biomaterials biocom- 
patibility and the macrophage. Biomaterials 5, 5-10.

Arvanitidou, M., Spaia, S., Katsinas, C. et al. (1998). Microbiological quality of water and dialysate in all haemodialysis centres of Greece. Nephrol. Dial. Transplant. 13, 949-954.

Association for the Advancement of Medical Instrumentation (2001). AAMI Standards and Recommended Practices, vol. 3 (1-332). Arlington: American National Standards Institute.

Atkins, E. and Morse, S. I. (1967). Studies in staphylococcal fever. VI. Responses induced by cell walls and various fractions of staphylococci and their products. Yale J. Biol. Med. 39, 297-311.

Atkins, E. and Huang, W.C. (1958). Studies on the pathogenesis of fever with influenza virus. I. The appearance of an endogenous pyrogen in the blood following intravenous injection of virus. J. Exp. Med. 107, 383-401.

Bambauer, R., Schauer, M., Jung, W. K. et al. (1994). Contamination of dialysis water and dialysate. A survey of 30 centers. ASAIO J. 40, 1012-1016.

Baz, M., Durand, C., Ragon, A. et al. (1991). Using ultrapure water in hemodialysis delays carpal tunnel syndrome. Int. J. Artif. Organs 14, 681-685.

Beeson, P. B. (1948). Temperature-elevating effect of a substance obtained from Polymorphonuclear leucocytes. J. Clin. Invest. 27, 524.

Bennett, I. L. and Beeson, P. B. (1953). Studies on the pathogenesis of fever. II. Characterization of fever-producing substances from polymorphonuclear leukocytes and from the fluid of sterile eluates. J. Exp. Med. 98, 493-508.

Blajchman, M. A., Beckers, E. A., Dickmeiss, E. et al. (2005). Bacterial detection of platelets: Current problems and possible resolutions. Transfus. Med. Rev. 19, 259-272.

Bodel, P. T. and Atkins, E. (1965). Studies in staphylococcal fever. V. Staphylococcal filtrate pyrogen. Yale J. Biol. Med. 38, 282-298.

Brandenburg, K., Howe, J., Gutsman, T. and Garidel, P. (2009). The expression of endotoxic activity in the Limulus test as compared to cytokine production in immune cells. Curr. Med. Chem. 16, 2653-2660.

Braude, A. I., McConnell, J. and Douglas, H. (1960). Fever from pathogenic fungi. J. Clin. Invest. 39, 1266-1276.

Brunson, K. W. and Watson, D. W. (1974). Pyrogenic specificity of streptococcal exotoxins, staphylococcal enterotoxin, and gram-negative endotoxin. Infect. Immun. 10, 347-351.

Carlin, G. and Viitanen, E. (2005). In vitro pyrogenicity of the diphtheria, tetanus and acellular pertussis components of a trivalent vaccine. Vaccine 23, 3709-3715.

Carlin, G. and Viitanen, E. (2003). In vitro pyrogenicity of a multivalent vaccine: Infanrix. PharmEuropa 15, 418-423.

Cinkotai, F. F., Lockwood, M. G. and Rylander, R. (1977). Airborne micro-organisms and prevalence of byssinotic symptoms in cotton mills. Am. Ind. Hyg. Assoc. J. 38, 554-559.

Daneshian, M., von Aulock, S. and Hartung, T. (2009). Assessment of pyrogenic contaminations with the validated human whole blood assay. Nature Protocols 12, 1709-1721.

Daneshian, M., Wendel, A., Hartung, T. and von Aulock, S. (2008). High sensitivity pyrogen testing in water and dialysis solutions. J. Immunol. Meth. 336, 64-70.

Daneshian, M., Guenther, A., Wendel, A. et al. (2006). In vitro pyrogen test for toxic or immunomodulatory drugs. J. Immunol. Meth. 313, 169-175.

De Boer, M., Reijneke, R., Van de Griend, R. J. et al. (1981). Large-scale purification and cryopreservation of human monocytes. J. Immunol. Methods 43, 225-239.

Dehus, O., Hartung, T. and Hermann, C. (2006). Endotoxin evaluation of eleven lipopolysaccharides by whole blood assay does not always correlate with Limulus amebocyte lysate assay. J. Endotoxin Res. 12, 171-180.

Deininger, S., Traub, S., Aichele, D. et al. (2008). Presentation of lipoteichoic acid potentiates its inflammatory activity. Immunobiol. 213, 519-529.

Dinarello, C. A. (2004). Infection, fever, and exogenous and endogenous pyrogens: some concepts have changed. J. Endotoxin Res. 10, 201-222.

Dinarello, C. A., Koch, K. M. and Shaldon, S. (1999). Interleukin-1 and its relevance in patients treated with hemodialysis. Kidney Int. 33, 21-26.

Dinarello, C. A., Cannon, J. G., Mancilla, J. et al. (1991). Interleukin-6 as an endogenous pyrogen: induction of prostaglandin E2 in brain but not in peripheral blood mononuclear cells. Brain Res. 562, 199-206.

Dinarello, C. A., Cannon, J. G., Wolff, S. M. et al. (1986). Tumor necrosis factor (cachectin) is an endogenous pyrogen and induces production of interleukin-1. J. Exp. Med. 163, 14331450 .

Dinarello, C. A., Koch, K. M. and Shaldon, S. (1988). Interleukin-1 and its relevance in patients treated with hemodialysis. Kidney Int. Suppl. 24, 21-26.

Dinarello, C. A., Goldin, N. P. and Wolff, S. M. (1974). Demonstration and characterization of two distinct human leukocytic pyrogens. J. Exp. Med. 139, 1369-1381.

Draing, C., Sigel, S., Deininger, S. et al. (2008). Cytokine induction by Gram-positive bacteria. Immunobiol. 213, 285-296.

Donham, K. J., Zavala, D. C. and Merchant, J. A. (1984a). Acute effects of the work environment on pulmonary functions of swine confinement workers. Am. J. Ind. Med. 5, 367-375.

Donham, K. J., Zavala, D. C. and Merchant, J. A. (1984b). Respiratory symptoms and lung function among workers in swine confinement buildings: a cross-sectional epidemiological study. Arch. Environ. Health 39, 96-101.

Emancipator, K., Csako, G. and Elin, R. J. (1992). In vitro inactivation of bacterial endotoxin by human lipoproteins and apolipoproteins. Infect. Immun. 60, 596-601.

Eperon, S. and Jungi, T. W. (1996). The use of human monocytoid lines as indicators of endotoxin. J. Immunol. Methods 194, 121-129.

Evans, R. C. and Holmes, C. J. (1991). In vitro study of the transfer of cytokine-inducing substances across selected highflux hemodialysis membranes. Blood Purif. 9, 92-101.

Favero, M. S., Petersen, N. J., Boyer, K. M. et al. (1974). Microbial contamination of renal dialysis systems and associated health risks. Trans. Am. Soc. Artif. Intern. Organs 20A, 175-183. 
Fennrich, S., Fischer, M., Hartung, T. et al. (1999). Detection of endotoxins and other pyrogens using human whole blood. Dev. Biol. Stand 101, 131-139.

Fischer, M., Keller-Stanislawski, B., Schober-Bendixen, S. et al. (2001). Effect of the preservative thiomersal on the release of interleukin-1 beta from human peripheral blood cells. $A L$ TEX 18, 47-49.

Grötsch, W., Leimbach, R. and Sonnenschein, B. (1992). On the safety of medical products: The detection of endotoxin on sterile surgical gloves. Hyg. Med. 17, 200-206.

Grötsch, W. and Eibach, F. (1990). Pyrogenprüfung von Verbandstoffen und verwandten Medicalprodukten. Hyg. Med. $15,45-54$.

Hartung, T. (2008). Food for thought ... on animal tests. ALTEX 25, 3-9.

Hartung, T., Aaberge, I., Berthold, S. et al. (2001). Novel pyrogen tests based on the human fever reaction. The report and recommendations of ECVAM workshop 43. ATLA 29, 99123.

Hartung, T. (2002). Comparison and validation of novel pyrogen tests based on the human fever reaction. ATLA 30 Suppl. 2, 49-51.

Hartung, T., Fennrich, S., Fischer, M. et al. (2000). Prevalidation of an alternative to the rabbit pyrogen test based on human whole blood. In M. Balls, A. M. van Zeller and M. E. Halder (eds.), Progress in the Reduction, Refinement and Replacement of Animal Experimentation (991-999). Amsterdam, The Netherlands: Elsevier Science B. V.

Hartung, T. and Wendel, A. (1995). Detection of Pyrogens using human whole blood. ALTEX 12, 70-75.

Hasiwa, M., Kullmann, K., von Aulock, S. et al. (2007). An in vitro pyrogen safety test for immune-stimulating components on surfaces. Biomaterials 28, 1367-1375.

Hoffmann, S., Peterbauer, A., Schindler, S. et al. (2005a). International validation of novel pyrogen tests based on human monocytoid cells. J. Immunol. Methods 298, 161-173.

Hoffmann, S., Luederitz-Puechel, U., Montag-Lessing, T. and Hartung, T. (2005b). Optimisation of pyrogen testing in parenterals according to different pharmacopoeias by probabilistic modelling. J. Endotoxin Res. 11, 25-31.

Hurton, L., Berkson, J. and Smith, S. (2009). Biology and Conservation of Horseshoe Crabs. The Effect of Hemolymph Extraction Volume and Handling Stress on Horseshoe Crab Mortality (331-346). Heidelberg, Springer-Verlag GmbH.

Kindinger, I., Daneshian, M., Baur, H. et al. (2005). A new method to measure air-borne pyrogens based on human whole blood cytokine response. J. Immunol. Methods 298, 143-153.

Klein, E., Pass, T., Harding, G. B. et al. (1990). Microbial and endotoxin contamination in water and dialysate in the central United States. Artif. Organs 14, 85-94.

Kobayashi, G. S. and Friedman, L. (1964). Characterization of the pyrogenicity of Candida albicans, saccharomyces cervisiae, and cryptococcus neoformans. J. Bacteriol. 88, 660666.

Kuehnert, M. J., Roth, V. R., Haley, N. R. et al. (2001). Transfusion-transmitted bacterial infection in the United States, 1998 through 2000. Transfusion 41, 1493-1499.

Kulander, L., Nisbeth, U., Danielsson, B. G. and Eriksson, O. (1993). Occurrence of endotoxin in dialysis fluid from 39 dialysis units. J. Hosp. Infect. 24, 29-37.

Kullmann, K. (2002). Adaption des In Vitro Pyrogen Tests (IPT) für prothetische Materialien, Master Thesis, Fachbereich Biologie der Universität Konstanz, Germany.

Kure, R., Grendahl, H. and Paulssen, J. (1982). Pyrogens from surgeons' sterile latex gloves. Acta Pathol. Microbiol. Immunol. Scand. [B] 90, 85-88.

Laburn, H. P., Rosendorff, C., Willies, G. and Woolf, C. (1974). Proceedings: A role for noradrenaline and cyclic AMP in prostaglandin E1 fever. J. Physiol. 240, 49P-50P.

Laurence, R. A. and Lapierre, S. T. (1995). Quality of hemodialysis water: a 7-year multicenter study. Am. J. Kidney Dis. $25,738-750$

Levin, J. and Bang, F. B. (1964a). A description of cellular coagulation in the limulus. Bull. Johns Hopkins Hosp. 115, 337345 .

Levin, J. and Bang, F. B. (1964b). The role of endotoxin in the extracellular coagulation of limulus blood. Bull. Johns Hopkins Hosp. 115, 265-274.

Li, S., Goorha, S., Ballou, L. R. and Blatteis, C. M. (2003). Intracerebroventricular interleukin-6, macrophage inflammatory protein-1 beta and IL-18: pyrogenic and PGE2-mediated? Brain Res. 992, 76-84.

Li, S., Ballou, L. R., Morham, S. G. and Blatteis, C. M. (2001). Cyclooxygenase- 2 mediates the febrile response of mice to interleukin-1beta. Brain Res. 910, 163-173.

Li, S., Wang, Y., Matsumura, K. et al. (1999). The febrile response to lipopolysaccharide is blocked in cyclooxygenase-2(-/-), but not in cyclooxygenase-1(-/-) mice. Brain Res. $825,86-94$.

Liebers, V., Stubel, H., Düser M. et al. (2009). Standardization of whole blood assay for determination of pyrogenic activity in organic dust samples. Int. J. Hygiene Env. Health 212, 547-556.

Lonnemann, G., Behme, T. C., Lenzner, B. et al. (1992). Permeability of dialyzer membranes to TNF alpha-inducing substances derived from water bacteria. Kidney Int. 42, 61-68.

Marion-Ferey, K., Leid, J. G., Bouvier, G. et al. (2005). Endotoxin level measurement in hemodialysis biofilm using "the whole blood assay". Artif. Organs 29, 475-481.

Mazzotti, F., Beuttler, J., Zeller, R. et al. (2007). In vitro pyrogen test - a new test method for solid medical devices. $J$. Biomed. Mater Res. 80, 276-282.

McClosky, W. T., Price, C. W., van Winkle, W. J. et al. (1943). Results of the first USP collaborative study of pyrogens. $J$. Am. Pharm. Assoc. 32, 69-73.

McDonald, C. P., Hartley, S., Orchard, K. et al. (1998). Fatal Clostridium perfringens sepsis from a pooled platelet transfusion. Transfus. Med. 8, 19-22.

Mitzner, S., Stange, J., Pichel, K. et al. (1995). Increased soluble CD14 levels in patients on hemodialysis. Influence of dialysate endotoxin or incompatibility to dialyzer membranes? ASAIO J 41, M707-708. 
Miyasaka, N., Sato, K., Kitano, Y. et al. (1992). Aberrant cytokine production from tenosynovium in dialysis associated amyloidosis. Ann. Rheum. Dis. 51, 797-802.

Mohr, L. C. (2004). Hypersensitivity pneumonitis. Curr. Opin. Pulm. Med. 10, 401-411.

Morath, S., Geyer, A., Spreitzer, I. et al. (2002). Structural decomposition and heterogeneity of commercial lipoteichoic acid preparation. Infect. Immun. 70, 938-944.

Morath, S., Geyer, A. and Hartung, T. (2001). Structure-function relationship of cytokine induction by lipotechoic acid from Staphylococcus aureus. J. Exp. Med. 193, 393-398.

Neal, P. A., Schneiter, R. and Caminita, B. H. (1942). Report on acute illness among rural mattress makers using low grade, stained cotton. JAMA 119, 1074-1082.

Pegues, D. A., Oettinger, C. W., Bland, L. A. et al. (1992). A prospective study of pyrogenic reactions in hemodialysis patients using bicarbonate dialysis fluids filtered to remove bacteria and endotoxin. J. Am. Soc. Nephrol. 3, 1002-1007.

Perez, P., Salmi, R., Folléa, G. et al. (2001). Determinants of transfusion-associated bacterial contamination: results of the French BACTHEM case-control study. Transfusion 41, 862871.

Peterbauer, A. and Werner-Felmayer, G. (1999). Further development of a cell culture model for the detection of bacterial pyrogens. ALTEX 16, 3-8.

Phillips, G., Hudson, S. and Stewart, W. K. (1994). Persistence of microflora in biofilm within fluid pathways of contemporary haemodialysis monitors (Gambro AK-10). J. Hosp. Infect. 27, 117-125.

Pool, E. J., Johaar, G., James, S. et al. (1998). The detection of pyrogens in blood products using an ex vivo whole blood culture assay. J. Immunoassay 19, 95-111.

Poole, S., Dawson, P. and Gaines Das, R. E. (1997). Second international standard for endotoxin: calibration in an international collaborative study. J. Endotoxin Res. 4, 221-231.

Prausnitz, C. (1936). Investigations on respiratory dust disease in operatives in the cotton industry. Medical Research Council, Special report series 212, 1-73, His Majesty's Stationery Office, London.

Raij, L., Shapiro, F. L. and Michael, A. F. (1973). Endotoxemia in febrile reactions during hemodialysis. Kidney Int. 4, 5760.

Rich, A. and Harris, A. K. (1981). Anomalous preference of cultured macrophages for hydrophobic and roughened substrata. J. Cell Sci. 50, 1-7.

Rietschel, E. T., Kirikae, T., Schade, F. U. et al. (1993). The chemical structure of bacterial endotoxin in relation to bioactivity. Immunobiol. 187, 169-190.

Refai, A. K., Textor, M., Brunette, D. M. and Waterfield, J. D. (2004). Effect of titanium surface topography on macrophage activation and secretion of proinflammatory cytokines and chemokines. J. Biomed. Res. 70A, 194-205.

Rosenberg, S. A. (2001). Cellular therapy: an introduction. Cancer J. 7 Suppl. 2, 51-52.

Rotta, J. (1975). Endotoxin-like properties of the peptidoglycan. Z. Immunitätsforsch. Exp. Klin. Immunol. 149, 230-244.
Rudloe, A. and Hernkind,A. E. (1983). The effect of heavy bleeding on mortality of the horseshoe crab, Limulus polyphemus, in the natural environment. J. Invertebr. Pathol. 42, 167-176.

Schindler, S., Spreitzer, I., Loschner, B. et al. (2006a). International validation of pyrogen tests based on cryopreserved human primary blood cells. J. Immunol. Meth. 316, 42-51.

Schindler, S., Rosenberg, U., Schlote, D. et al. (2006b). Pyrogen testing of lipidic parenterals with a novel in vitro test. PharmEuropa Sci. Notes, 1-7.

Schindler, S., Asmus, S., von Aulock, S. et al. (2004). Cryopreservation of human whole blood for pyrogenicity testing. $J$. Immunol. Meth. 294, 89-100.

Schindler, S., Bristow, A., Cartmell, T. et al. (2003). Comparison of the reactivity of human and rabbit blood towards pyrogenic stimuli. ALTEX 20, 59-63.

Schleifer, K. H. (1975). Chemical structure of the peptidoglycan, its modifiability and relation to the biological activity. Z. Immunitätsforsch. Exp. Klin. Immunol. 149, 104-117.

Schouten, W. E., Grooteman, M. P., van Houte, A. J. et al. (2000). Effects of dialyser and dialysate on the acute phase reaction in clinical bicarbonate dialysis. Nephrol.Dial.Transplant. 15, 379-384.

Schwalbe, S., Holzhauer, M., Schaeffer, J. et al. (1997). Beta 2-microglobulin associated amyloidosis: a vanishing complication of long-term hemodialysis. Kidney Int. 52, 10771083 .

Shmunes, E. and Darby, T. (1984). Contact dermatitis due to endotoxin in irradiated latex gloves. Contact Dermatitis 10, 240-244.

Soskolne, W. A., Cohen, S., Sennerby, L. et al. (2002). The effect of titanium surface roughness on the adhesion of monocytes and their secretion of TNF- $\alpha$ and PGE2. Clin. Oral Implants Res. 13, 86-93.

Spreitzer, I., Löschner, B., Schneider, C. K. et al. (2007). 10 years of experience with alternative pyrogen tests (monocyte activation tests). AATEX 14 Spec. Issue, 587-589. Proc. 6th World Congress on Alternatives \& Animal Use in the Life Sciences August 21-25, 2007, Tokyo, Japan.

Spreitzer, I., Fischer, M., Hartzsch, K. et al. (2002). Comparative study of rabbit pyrogen test and human whole blood assay on human serum albumin. ALTEX 19 Suppl. 1,73-75.

Steere, A. C., Rifaat, M. K., Seligmann, E. B. et al. (1978). Pyrogen reactions associated with the infusion of normal serum albumin (human). Transfusion 18, 102-107.

Stoddard, M. B., Pinto, V., Keiser, P. B. and Zollinger, W. (2009). Evaluation of a Whole Blood Cytokine Release Assay for Use in Measuring Endotoxin Activity of Group B Neisseria meningitidis Vaccines Made from Lipid A Acylation Mutants. Clin. Vaccine Immunol, published online ahead of print on 18 November 2009.

Tranaeus, A. and Yao, Q. (2008). Immune dysfunction in dialysis patients - prevention and treatment strategies. Perit. Dial. Int. 28 Suppl. 3, S161-166.

Thelin, A., Tegler, O. and Rylander, R. (1984). Lung reactions during poultry handling related to dust and bacterial endotoxin levels. Eur. J. Respir. Dis. 65, 266-271. 
Thompson, M. (1998). Assessments of the population biology and critical habitat for the horseshoe crab, Limulus polyphemus, in the South Atlantic Bight. Master's thesis 1998, Medical University of South Carolina, University of Charleston.

Tsuchida, K., Takemoto, Y., Yamagami, S. et al. (1997). Detection of peptidoglycan and endotoxin in dialysate, using silkworm larvae plasma and limulus amebocyte lysate methods. Nephron 75, 438-443.

Urena, P., Herbelin, A., Zingraff, J. et al. (1992). Permeability of cellulosic and non-cellulosic membranes to endotoxin subunits and cytokine production during in vitro haemodialysis. Nephrol.Dial. Transplant. 7, 16-28.

Ushikubi, F., Segi, E., Sugimoto, Y. et al. (1998). Impaired febrile response in mice lacking the prostaglandin $\mathrm{E}$ receptor subtype EP3. Nature 395, 281-284.

Walls, E. A. and Berkson, J. (2003). Effects of blood extraction on horseshoe crabs (Limulus polyphemus). Fish Bull 101, 457-459.

Ward, R. A. (2004). Ultrapure dialysate. Semin. Dial. 17, 489497.

Watson, D. W. (1960). Host-parasite factors in group A streptococcal infections: pyrogenic and other effects of immunologic distinct exotoxins related to scarlet fever toxins. J. Exp. Med. 111, 255-284.

Welsh, H., Calvery, H. O., McClosky, W. T. and Price, C. W. (1943). Method of Preparation and Test for Bacterial Pyrogen. J. Am. Pharm. Assoc. 3, 65-69.

Werner, L., Tetz, M., Mentak, K. et al. (2009). Detection of pyrogens adsorbed to intraocular lenses - Evaluation of limulus amoebocyte lysate and in vitro pyrogen tests. Journal Cataract \& Refract Surg. 35, 1273-1280.

Werner-Felmayer, G., Baier-Bitterlich, G., Fuchs, D. et al. (1995). Detection of Bacterial Pyrogens on the Basis of their Effects on Gamma Interferon-Mediated Formation of Neopterin or Nitrite in Cultured Monocyte Cell Lines. Clin. Diagn. Lab. Immunol. 2, 307-313.

Wollowitz, S. (2001). Fundamentals of the psoralen-based
Helinx technology for inactivation of infectious pathogens and leukocytes in platelets and plasma. Semin. Hematol. 38 Suppl. 11, 4-11.

Ziats, N. P., Miller, K. M. and Anderson, J. M. (1988). In vitro and in vivo interactions of cells with biomaterials. Biomaterials 9, 5-13.

Zimmermann. M., Busch, K., Kuhn, S. and Zeppezauer, M. (1999). Endotoxin adsorbent based on immobilized human serum albumin. Clin. Chem. Lab. Med. 37, 373-379

Zucker, B. A., Draz, A. and Müller, W. (2000). Comparison of filtration and impingement for sampling airborne endotoxin. J. Aerosol. Sci. 31, 751-755.

\section{Acknowledgements}

The validation study was funded by the European Union (QLRT-1999-00811).

The authors would like to thank the numerous scientific and industrial partners in 15 years of development, but especially Dr. Thomas Montag, Dr. Ingo Spreitzer, Paul-Ehrlich-Institut Langen and Stefan Fennrich, now NMI Reutlingen for their long lasting collaboration and friendship. The technology is protected by three granted patents in the EU (EP0851231, July $1^{\text {st }}, 1998$, EP0741294, November $\left.6^{\text {th }} 1996\right)$, the U.S. (5 891 728, May $2^{\text {nd }}, 1996$ ), and Japan (Nr. 8137 713, July, 6, 2005, Nr. 4291580, April 10 ${ }^{\text {th }} 2009$ ).

\section{Correspondence to}

Thomas Hartung, MD, PhD

Center for Alternatives to Animal Testing (CAAT)

Johns Hopkins University

Bloomberg School of Public Health

615 N. Wolfe St.

Baltimore, MD, 21205

e-mail: thartung@jhsph.edu

Tel: +1 4106144990

Fax: +1 4106142871 